\title{
Boston University-Boston Medical Center Cancer Center
}

National Cancer Institute

\section{Source}

National Cancer Institute. Boston University-Boston Medical Center Cancer Center. NCI Thesaurus. Code C157454.

A cancer center with the mission of providing a bridge between the clinical oncology and biomedical research communities at the Boston University Medical and Charles River campuses and the Boston Medical Center. 